\title{
Modelling the short-term and long-term aggregate response of multiple loads fed through a sub-transmission network
}

\author{
Thierry Van Cutsem, Fellow, IEEE
}

\author{
Quentin Renoy
}

\author{
Daniel Lefebvre
}

\begin{abstract}
This paper addresses the problem of representing the aggregate response to voltage changes of a set of loads fed through distribution transformers connected by a subtransmission network. A simple, while accurate equivalent is proposed. The latter involves standard network components and includes a single internal bus. This equivalent matches both the short-term and the long-term response of the original system, the long-term dynamics coming from the load tap changers that control the distribution voltages. A procedure to identify its parameters from step responses of the unreduced system is detailed. The method is illustrated and validated on a large subtransmission-distribution system of Hydro-Québec.
\end{abstract}

Index Terms-Load modelling, equivalent, sub-transmission, distribution, short-term dynamics, long-term dynamics, load tap changers

\section{INTRODUCTION}

$\mathbf{I}$ $\mathrm{T}$ is widely agreed that better models are needed for simulating the response of loads to disturbances affecting the transmission system. This is of paramount importance in voltage stability studies where load dynamics play an important rôle [1]. Indeed, load power restoration is one of the driving force of voltage instability [2].

Considerable attention has been paid to deriving practical models of either individual or composite loads at distribution level [3], [4], [5]. Further efforts should be also devoted to improving load models at the transmission level [6], [7]. This requires a better representation of the aggregate response of the downstream loads together with the sub-transmission networks (and their shunt compensation) and the transformers feeding the distribution buses. The latter are usually equipped with Load Tap Changers (LTCs) aimed at controlling the distribution voltages.

The motivation for replacing those parts of the system by a simple model is twofold. First, a significant decrease of the model complexity can be obtained by eliminating numerous buses at lower voltage levels. The second reason is the practical unavailability of real-time data at the sub-transmission and distribution levels in the control centers of Transmission System Operators (TSOs), where voltage stability studies are

T. Van Cutsem (t.vancutsem@ulg.ac.be) is with the Belgian National Fund for Scientific Research (FNRS) at the Dept. of Electrical Engineering and Computer Science (Montefiore Institute) of the University of Liège, Sart Tilman B37, B-4000 Liège, Belgium. Q. Renoy is a final-year student in Electrical Engineering at the same university. D. Lefebvre (lefebvre.daniel.4@hydro.qc.ca) is with the TransEnergie division of Hydro-Québec, Complexe Desjardins, Tour Est - 10ème étage, CP 10000, Montréal (QC) H5B1H7, Canada. performed. These parts of the system are usually unobservable by the TSO state estimator, which can only determine the power leaving the transmission system (i.e. entering the system to be replaced by an equivalent). Thus, measurements have to be collected off-line or some assumptions have to be made, in order to expand the model down to the lower voltage levels. In such conditions, keeping the sub-transmission and distribution buses in the model is not likely to be more accurate than an aggregate response model.

Generic models of load power restoration have been proposed for some time, either in additive [8], [9], [10] or in multiplicative form [2]. While these models are suitable to account for the load power restoration taking place in thermostatically controlled loads, their use for modelling the above mentioned aggregate load response is questionable. Indeed, these models involve a continuous-time dynamics that does not account for the discrete behaviour of LTCs (i.e. step changes in ratio occuring after various pure delays). Furthermore, as they focus on the power flow at the "entry point" of the system, these models cannot be easily updated with respect to internal changes, such as variations of the demand and/or compensation at distribution level.

On the contrary, this paper focuses on an equivalent that retains the nature of the original system, while preserving simplicity of use and offering good accuracy.

The proposed method is being tested on various subtransmission-distribution networks of the Hydro-Québec system. This application is described and preliminary test results are reported.

The paper is organized as follows. The problem is stated in Section II. The chosen equivalent is described and the identification of its parameters from step responses of the unreduced system is presented in Section III. This procedure is illustrated in some detail on a real-life example and validation tests are reported in Section IV. Some concluding remarks are given in Section V.

\section{STATEMENT OF THE PROBLEM}

The type of system to be replaced by an equivalent is sketched with solid lines in Fig. 1. In the general case, it includes:

- High Voltage (HV) overhead lines or cables

- transformers feeding Medium Voltage (MV) distribution buses. The latter are equipped with LTCs with different delays before the first and the subsequent tap changes 
- shunt capacitors at HV and/or MV levels

- loads connected to MV buses

- distributed generation at MV buses (operating under constant power factor or somewhat participating to voltage control).

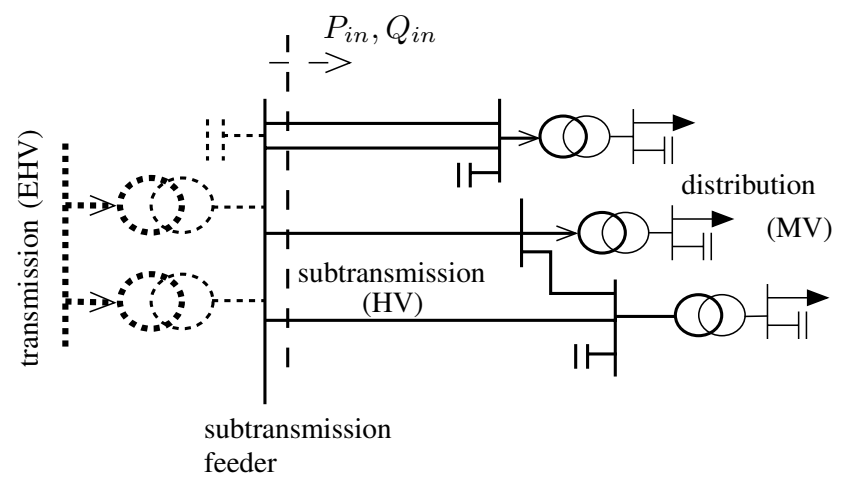

Fig. 1. System to be replaced by equivalent (shown with solid lines)

Note that the system to be reduced may be meshed (as in Fig. 1). On the other hand, it is assumed to be fed through a single HV bus, which is called sub-transmission feeder in the sequel. Sub-transmission systems with multiple feeders are not considered in this paper.

Note also that the system to be replaced by an equivalent does neither include the transformer(s) through which it is connected to the transmission grid, nor the shunt compensation connected to the feeder bus, all shown with dotted lines in Fig. 1. These components are thus retained explicitly in the final model.

The sought equivalent should meet the following requirements:

1) it should match as closely as possible the response of the original system to a variation of the sub-transmission feeder voltage. Thus, following a disturbance in the transmission system, the active power $P_{i n}$ and the reactive power $Q_{i n}$ entering the system (see Fig. 1) should be the same:

- at the short-term equilibrium point reached after electromechanical transients have died out, but before any LTC has responded

- at the long-term (final) equilibrium reached after all LTCs have acted

2) the equivalent should be as simple as possible

3) it should have a physical interpretation

4) its parameters should be determined automatically from responses of the original system

5) the equivalent should be accurate for a reasonably large variations of the load level. Such changes are considered in the determination of security limits

6) the equivalent should accommodate various individual load models.

In this work we have considered loads modelled under the quasi steady-state approximation [2], i.e. with their dynamics neglected and replaced by equilibrium conditions. Hence, the inertial response of motors is not considered here, although it would not make a problem to handle a dynamic model of the motors.

Thus, we assume a variation of the active power $P_{\ell i}$ and reactive power $Q_{\ell i}$ with the voltage $V_{i}$ at bus $i$ given by the multi-exponential model:

$$
\begin{array}{rlr}
P_{\ell i} & =P_{\ell i}^{o} \sum_{j=1}^{n_{i}} a_{i j} V_{i}^{\alpha_{i j}} & \text { with } \\
Q_{\ell i}=Q_{\ell i}^{o} \sum_{j=1}^{n_{i}} b_{i j} V_{i}^{\beta_{i j}} & \text { with } \sum_{j=1}^{m_{i}} b_{i j}=1
\end{array}
$$

The $a_{i j}$ and $\alpha_{i j}$ coefficients can be obtained by fitting the model (1) in the least-square sense to a set of $\left(V_{i}, P_{\ell i}\right)$ points obtained from a detailed model of the load [5]. The $b_{i j}$ and $\beta_{i j}$ coefficients are obtained in the same way from $\left(V_{i}, Q_{\ell i}\right)$ points.

\section{THE LOAD EQUIVALENT AND ITS IDENTIFICATION}

\section{A. Structure of the load equivalent}

Figure 2 shows the equivalent to be considered. All individual loads in the system are merged into a single load $\left(P_{\ell}, Q_{\ell}\right)$. Thus, if there are $c$ individual loads, each modelled by $(1,2)$, one has:

$$
\begin{array}{ll}
P_{\ell}\left(V_{c}\right)=\sum_{i=1}^{c} P_{\ell i}=\sum_{i=1}^{c} P_{\ell i}^{o} \sum_{j=1}^{n_{i}} a_{i j} V_{c}^{\alpha_{i j}} \quad \text { with } \sum_{j=1}^{n_{i}} a_{i j}=1 \\
Q_{\ell}\left(V_{c}\right)=\sum_{i=1}^{c} Q_{\ell i}=\sum_{i=1}^{c} Q_{\ell i}^{o} \sum_{j=1}^{m_{i}} b_{i j} V_{c}^{\beta_{i j}} & \text { with } \sum_{j=1}^{m_{i}} b_{i j}=1
\end{array}
$$

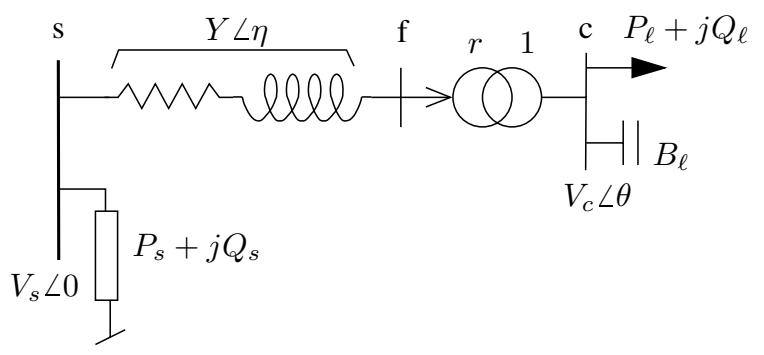

Fig. 2. Proposed equivalent

Similarly, all the shunt capacitors connected to MV bus are merged into a single equivalent shunt $B_{\ell}$.

An ideal transformer with ratio $r$ dynamically controls the voltage at bus $c$, thus reproducing the voltage control by the corresponding HV-MV transformers.

Finally, the series admittance $Y \angle \eta$ and the power injection $P_{s}+j Q_{s}$ account for the sub-transmission system. We take constant $Y$ and $\eta$ for simplicity. On the other hand, a voltage dependent injection is considered, with again the multiexponential model:

$$
P_{s}=P_{s}^{o} \sum_{j=1}^{n_{s}} a_{j} V_{s}^{\alpha_{j}} \quad \text { with } \quad \sum_{j=1}^{n_{s}} a_{j}=1
$$




$$
Q_{s}=Q_{s}^{o} \sum_{j=1}^{m_{s}} b_{j} V_{s}^{\beta_{j}} \quad \text { with } \sum_{j=1}^{m_{s}} b_{j}=1
$$

where $V_{s}$ is the voltage at bus the feeder bus $s$.

\section{B. Principle of the identification}

The parameters of the equivalent are identified so as to match the response of the original system to voltage variations. To this purpose, an ideal voltage source is connected to the sub-transmission feeder, as shown in Fig. 3, and step variations of the voltage are considered. The time evolution of the active power $P_{i n}$ and reactive power $Q_{i n}$ entering the system is simulated for further reference.

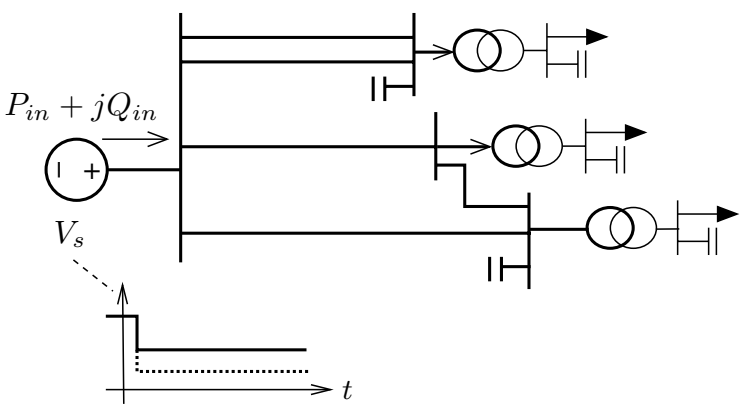

Fig. 3. Simulating the response of the original system

A typical time evolution of $P_{i n}$ to be matched by the equivalent is shown in Fig. 4. The curve has been obtained on the real system further considered in Section IV, subjected to a $0.1 \mathrm{pu}$ decrease of $V_{s}$ at $t=2 \mathrm{~s}$. As expected, the power drops under the effect of load sensitivity to voltage, and then recovers under the effect of LTCs.

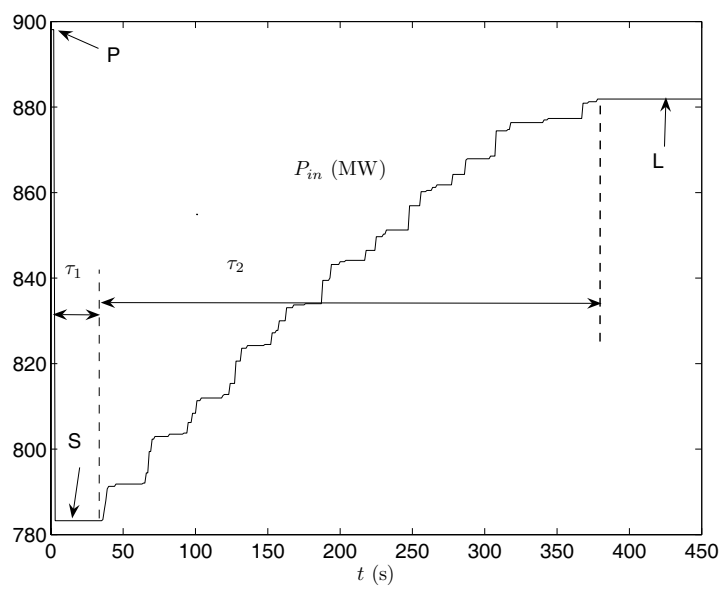

Fig. 4. Typical step response to be matched by equivalent

The identification is performed in three steps, as detailed in the next three subsections.

\section{Step 1: identifying the series admittance}

The first step consists of identifying the magnitude $Y$ and phase angle $\eta$ of the series admittance.
Let $V_{c} \angle \theta$ be the complex voltage at bus $c$, the voltage at bus $s$ being taken as phase angle reference. The active and reactive power balance equations at bus $s$ can be written as:

$$
\begin{aligned}
& P_{s}+V_{s}^{2} Y \cos \eta-r V_{s} V_{c} Y \cos (\theta-\eta)-P_{i n}=0 \\
& Q_{s}-V_{s}^{2} Y \sin \eta-r V_{s} V_{c} Y \sin (\theta-\eta)-Q_{i n}=0
\end{aligned}
$$

and similarly at bus $c$ :

$$
\begin{gathered}
r^{2} V_{c}^{2} Y \cos \eta-r V_{s} V_{c} Y \cos (-\theta+\eta)+P_{\ell}\left(V_{c}\right)=0 \\
-V_{c}^{2} r^{2} Y \sin \eta-r V_{s} V_{c} Y \sin (-\theta+\eta)+Q_{\ell}\left(V_{c}\right)-B_{\ell} V_{c}^{2}=0
\end{gathered}
$$

These four equations can be rewritten in compact vector form as:

$$
\mathbf{f}\left(P_{s}, Q_{s}, V_{c}, \theta, r, Y, \eta, V_{s}, P_{i n}, Q_{i n}\right)=\mathbf{0}
$$

with $\mathrm{f}: \mathcal{R}^{10} \rightarrow \mathcal{R}^{4}$. Note that $V_{s}, P_{i n}$ and $Q_{i n}$ are not shown in (11) since their values are known, being either chosen or provided by the time simulation of the unreduced system.

With reference to Fig. 4, the above equations hold at respectively the pre-contingency operating $\mathrm{P}$, the short-term equilibrium point $\mathrm{S}$ and the long-term point equilibrium $\mathrm{L}$ identified on the figure. This can be written in vector form as:

$$
\begin{array}{r}
\mathbf{f}\left(P_{s}^{p}, Q_{s}^{p}, V_{c}^{p}, \theta^{p}, r^{p}, Y, \eta, V_{s}^{p}, P_{i n}^{p}, Q_{i n}^{p}\right)=\mathbf{0} \\
\mathbf{f}\left(P_{s}^{s}, Q_{s}^{s}, V_{c}^{s}, \theta^{s}, r^{s}, Y, \eta, V_{s}^{s}, P_{i n}^{s}, Q_{i n}^{s}\right)=\mathbf{0} \\
\mathbf{f}\left(P_{s}^{l}, Q_{s}^{l}, V_{c}^{l}, \theta^{l}, r^{l}, Y, \eta, V_{s}^{l}, P_{i n}^{l}, Q_{i n}^{l}\right)=\mathbf{0}
\end{array}
$$

where the upperscripts $p, s$ and $l$ refer to points $\mathrm{P}, \mathrm{S}$ and $\mathrm{L}$, respectively. As detailed in Table I, some variables appearing in (12-14) have known values and some others have identical values. Indeed:

- $P_{s}$ depends on $V_{s}$ only; since $V_{s}$ remains unchanged when passing from $\mathrm{S}$ to $\mathrm{L}$ (step signal) so does $P_{s}$

- the same holds true for $Q_{s}$

- points $\mathrm{P}$ and $\mathrm{L}$ are steady-state equilibria, where the voltage $V_{c}$ controlled by the LTC is taken equal to its setpoint value $V_{c}^{o}$ (neglecting deadband effects)

- the ratio $r$ has the same value at points $\mathrm{S}$ and $\mathrm{L}$ since the LTC has not acted yet.

Only 10 variables remain unknown, identified by the question marks in Table I. Together with $Y$ and $\eta$, this leads to eventually 12 unknowns, balanced by the $3 \times 4=12$ equations (12-14). A nonlinear solver is used to solve the latter.

TABLE I

PARAMETERS TO BE COMPUTED

\begin{tabular}{|c||c|c|c|}
\hline variable & at point $\mathrm{P}$ & at point $\mathrm{S}$ & at point $\mathrm{L}$ \\
\hline$P_{s}$ & $P_{s}^{p}=?$ & $P_{s}^{s}=?$ & $P_{s}^{l}=P_{s}^{s}$ \\
$Q_{s}$ & $Q_{s}^{p}=?$ & $Q_{s}^{s}=?$ & $Q_{s}^{l}=Q_{s}^{s}$ \\
$V_{c}$ & $V_{c}=V_{c}^{o}$ & $V_{c}^{s}=?$ & $V_{c}=V_{c}^{o}$ \\
$\theta$ & $\theta^{p}=?$ & $\theta^{s}=?$ & $\theta^{l}=?$ \\
$r$ & $r^{p}=?$ & $r^{s}=r^{p}$ & $r^{l}=?$ \\
\hline
\end{tabular}




\section{Step 2: identifying the equivalent transformer dynamics}

A discrete LTC is assumed for the equivalent. The next step consists of determining the delays affecting the initial and subsequent tap changes, when the controlled voltage $V_{c}$ leaves the deadband $\left[V_{c}^{o}-\epsilon V_{c}^{o}+\epsilon\right]$ assumed for the LTC.

It is easily identified from Fig. 4 that the load power restoration by the LTCs starts $\tau_{1}$ seconds after the disturbance, and is completed $\tau_{2}$ seconds later. Note that not all LTCs start, move and stop at the same time and hence the rate of change of power is not constant. The equivalent is aimed at reproducing the average slope of this variation.

To this purpose, the equivalent LTC should have a delay $\tau_{1}$ on its first move and it should decrease the ratio $r$ from $r^{p}$ (value at point $\mathrm{S}$ ) to $r^{l}$ (value at point $\mathrm{L}$ ) in $\tau_{2}$ seconds, which corresponds to a rate of change:

$$
\rho=\frac{r^{p}-r^{l}}{\tau_{2}}
$$

Alternatively $\rho$ could be obtained as the slope of a straight line fitted, in the least-square sense, to the system response over the recovery period.

Assuming furthermore that the ratio $r$ changes by $\Delta r$ when the equivalent LTC moves by one position, the delay in between two tap changes is given by $\Delta r / \rho$. The choice of $\Delta r$ is free but it should be small enough in order the equivalent to have a smooth response.

$\epsilon$ can be taken as the average half-deadband of all LTCs.

\section{E. Step 3: modelling the variation of $P_{s}$ and $Q_{s}$ with $V_{s}$}

The last step consists of determining the parameters $a_{j}, \alpha_{j}, b_{j}, \beta_{j}$ of the model $(5,6)$ relating powers $P_{s}$ and $Q_{s}$ to voltage $V_{s}$.

To this purpose, the short-term equilibrium point $\mathrm{S}$ (see Fig. 4) of the unreduced system is determined for $v$ different step variations of $V_{s}$. Let us denote by $\left(P_{i n}\right)_{i}+j\left(Q_{i n}\right)_{i}$ the complex power entering the system when the source voltage is set to $\left(V_{s}\right)_{i} \quad(i=1, \ldots, v)$.

The corresponding values of $\left(P_{s}\right)_{i}$ and $\left(Q_{s}\right)_{i}$ can be easily obtained from Eqs. (7-10) in which $Y, \eta, V_{s}, P_{i n}$ and $Q_{i n}$ are replaced by their known values and $r$ is set to $r^{p}$. To this purpose, Eqs. $(9,10)$ are first solved for $V_{c}$ and $\theta$, and the latter are introduced into $(7,8)$ to obtain $P_{s}$ and $Q_{s}$.

The load model (5) is then fitted to the computed points $\left(V_{s}\right)_{i},\left(P_{s}\right)_{i}(i=1, \ldots, v)$ in the least-square sense:

$\min _{P_{s}^{o}, a_{j}, \alpha_{j}} \sum_{i=1}^{v}\left(\left(P_{s}\right)_{i}-P_{s}^{o} \sum_{j=1}^{n_{s}} a_{j}\left(V_{s}\right)_{i}^{\alpha_{j}}\right)^{2}$ with $\sum_{j=1}^{n_{s}} a_{j}=1$

and similarly for the reactive power:

$$
\min _{Q_{s}^{o}, b_{j}, \beta_{j}} \sum_{i=1}^{v}\left(\left(Q_{s}\right)_{i}-Q_{s}^{o} \sum_{j=1}^{m_{s}} b_{j}\left(V_{s}\right)_{i}^{\beta_{j}}\right)^{2} \text { with } \sum_{j=1}^{m_{s}} b_{j}=1 .
$$

\section{ILLUSTRATIVE EXAMPLE}

\section{A. System description}

The proposed method is illustrated on the system shown in Fig. 5, which is part of the Hydro-Québec network and serves a large load in the Montréal area.

The sub-transmission feeder (bus B1228) is fed from the $315-\mathrm{kV}$ transmission bus (B428) by three transformers in parallel, and has four shunt capacitors connected, each of 92.5 Mvar. Let us recall that these equipments are not included in the system to be equivalenced, and will be connected to the equivalent in the final model.

The network includes 14 sub-transmission 120-kV lines and cables, 29 transformers feeding the 12.9 and $26.4-\mathrm{kV}$ distribution buses, and 9 shunt capacitors at distribution level. All these transformers are equipped with LTCs having delays in the range [33 65] seconds for the first tap change and [12 60] seconds for the subsequent changes.

The initial power balance of the system (provided by a load flow computation) is detailed in Fig. 5. It can be seen that the reactive power losses are significant compared to the reactive load.

All individual loads are represented by an exponential model, i.e. $(1,2)$ with $n_{i}=m_{i}=1$. Unless otherwise specified, $\alpha_{1}$ and $\beta_{1}$ have been set to 1.3 and 2.0, respectively, in accordance with a Hydro-Québec practice. Let us stress that load models with more terms can be easily used, to better fit the behaviour of composite loads [5].

Note that the loads at buses B7012 and B7026, which amount to $3 \%$ of the total load power, are not controlled by LTCs. Nevertheless, they have been merged with the other loads behind the equivalent LTC.

Quasi steady-state time simulation [2] has been used in this study, since motor inertia effects are not considered. Any other long-term simulation method would be appropriate.

\section{B. Identification of the equivalent parameters}

1) Step 1: The response of the original system to a voltage drop of 0.10 pu was used to identify $Y$ and $\eta$. The corresponding evolution of the input active power $P_{i n}$ has been shown in Fig. 4, while the corresponding reactive power $Q_{i n}$ is given in Fig. 6. The latter evolves quite differently. In the short term, under the effect of the voltage drop, the load consumption decreases. However, the reactive network losses increase and the production of shunt capacitors decreases so much that $Q_{i n}$ slightly increases. When the load power is restored by the LTCs, this effect is even more pronounced.

Solving the pre-contingency, short-term and long-term equations (12-14) yields a series impedance $Y^{-1} e^{-j \eta}=0.0016+$ j0.0073 pu on a 100-MVA base.

2) Step 2: The delay on the first tap change of the equivalent LTC was set to $33 \mathrm{~s}$ while the rate of change of the ratio was found to be $2.9610^{-4} \mathrm{~s}^{-1}$. Chosing $\Delta r=0.006 \mathrm{pu}$, the delay in between two tap changes was set to $20.3 \mathrm{~s}$.

3) Step 3: To obtain the various short-term equilibria, the response of the original system to a ramp variation of $V_{s}$ was determined, with all LTCs blocked. $v=40$ points have been 


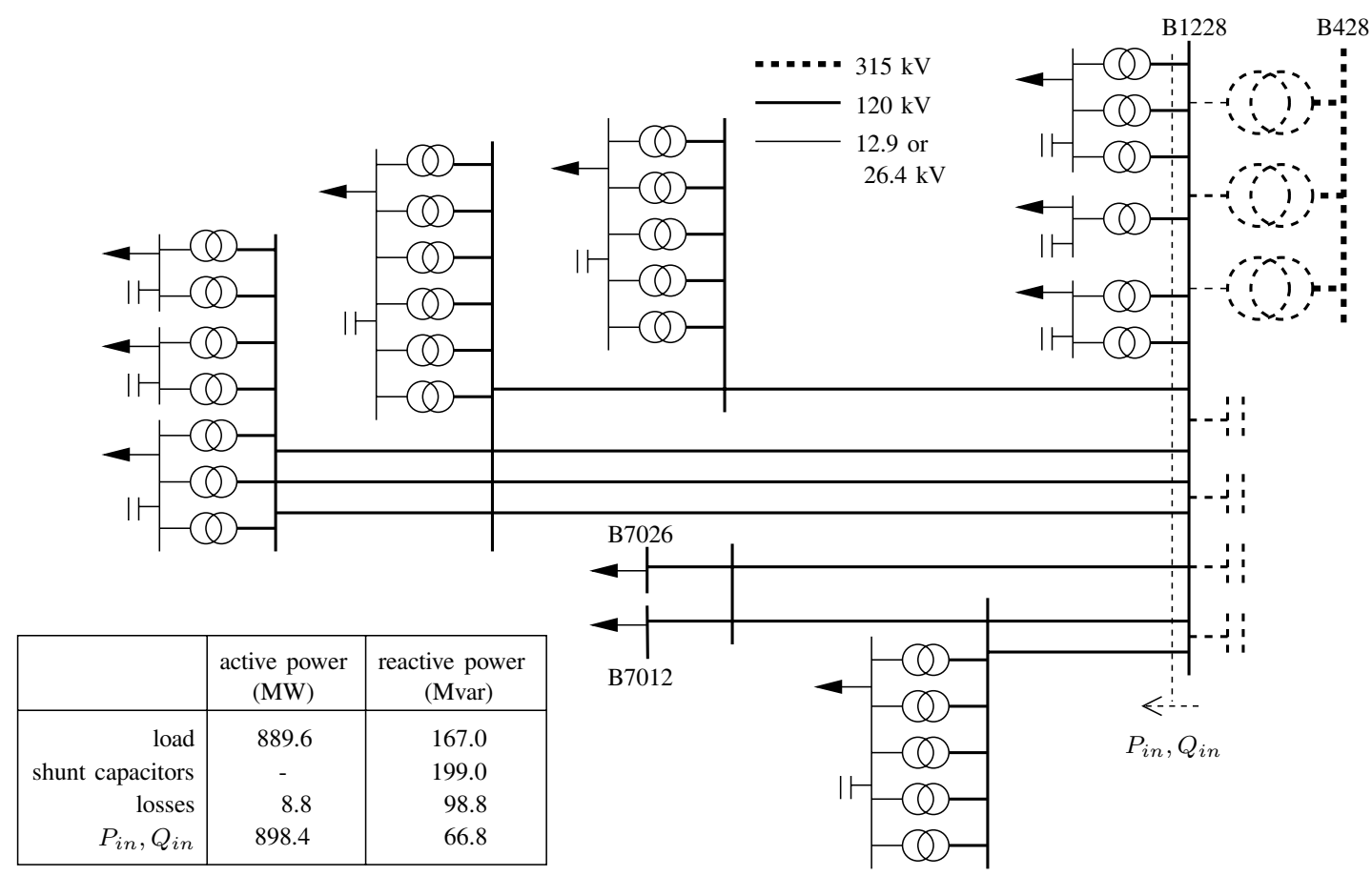

Fig. 5. System used for testing the method

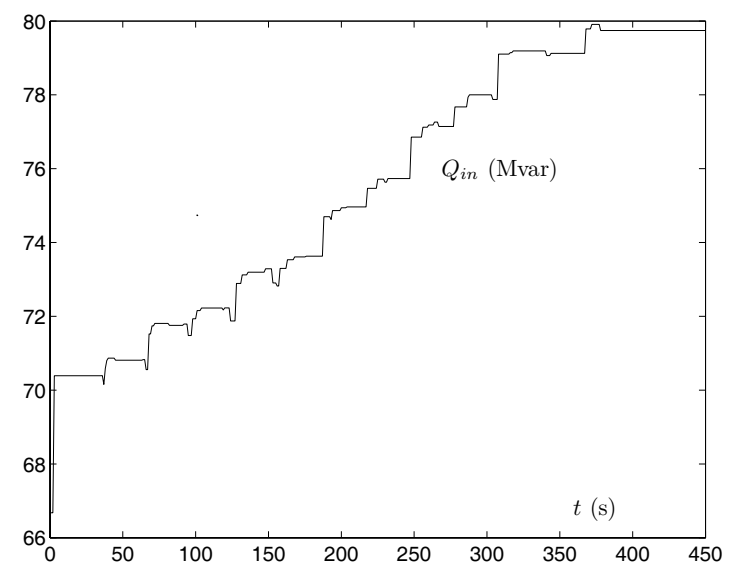

Fig. 6. Response of original system to voltage drop: reactive power

collected for a variation of $V_{s}$ from -0.30 to +0.15 pu around its base case value.

The relatively small changes in active power losses allows to take a single term in (5) while two terms are preferred in (6) to reproduce the relatively larger changes in reactive power. The least-square adjustment according to $(15,16)$ yields the following model:

$$
\begin{aligned}
P_{s} & =-0.038 V_{s}^{-2.09} \\
Q_{s} & =0.407\left(1.968 V_{s}^{0.726}-0.968 V_{s}^{1.834}\right)
\end{aligned}
$$

in per unit on a 100-MVA base.

\section{Validation of the equivalent}

The first validation consists in comparing the responses of the original and equivalent systems to the $0.1 \mathrm{pu}$ voltage drop already considered at Step 1 of the identification. This comparison is provided in Fig. 7. Expectedly, the equivalent is a very good approximation of the original system. The powers at the short and long-term equilibria are the same, as required by the method.

Since $Y$ and $\eta$ were determined from the response to a single voltage drop, the validity of the equivalent had to be checked for other voltage variations. For instance, Fig. 8 shows the responses of the original and equivalent systems to a $0.04 \mathrm{pu}$ voltage drop. The final discrepancy observed for the active power is attributable to the deadband of the equivalent LTC. The fact that various distribution voltages were not exactly restored to their setpoint values is also responsible for the incomplete active power restoration that can be observed in Figs. 7 and 8. It seems difficult to better account for this distributed deadband effect in the equivalent. In any case, the final error is quite acceptable ( $3 \mathrm{MW}$ for an inital power of $898 \mathrm{MW})$.

The response of the equivalent has been also tested in the presence of the upper-level, $315 / 120-\mathrm{kV}$ transformers, which are also equipped with LTCs. To this purpose, the transformers and the shunt compensation were connected to the subtransmission feeder, and the voltage source was attached to the $315-\mathrm{kV}$ transmission bus. The response to a $0.1 \mathrm{pu}$ decrease of this voltage is given in Fig. 9, which shows the total active and reactive power flows in the above mentioned transformers. The reactive power flow is much influenced by the large 

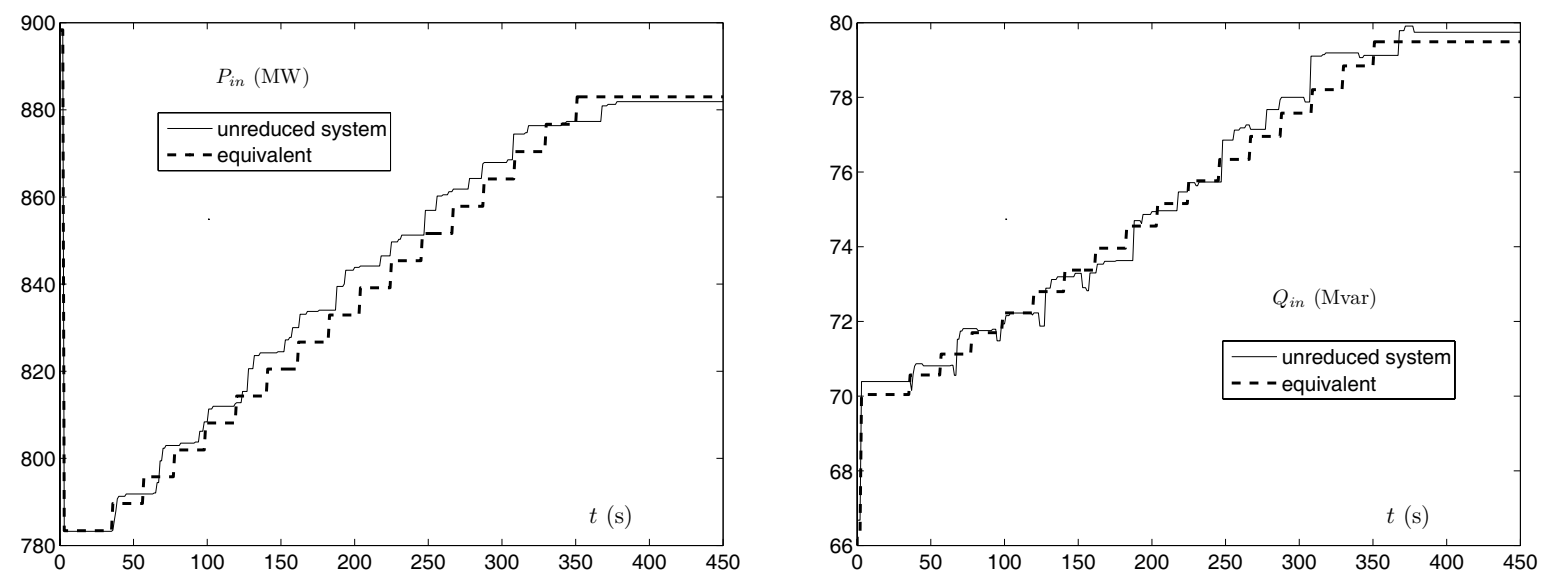

Fig. 7. active and reactive power evolution following a $0.1 \mathrm{pu}$ voltage drop at the subtransmission feeder
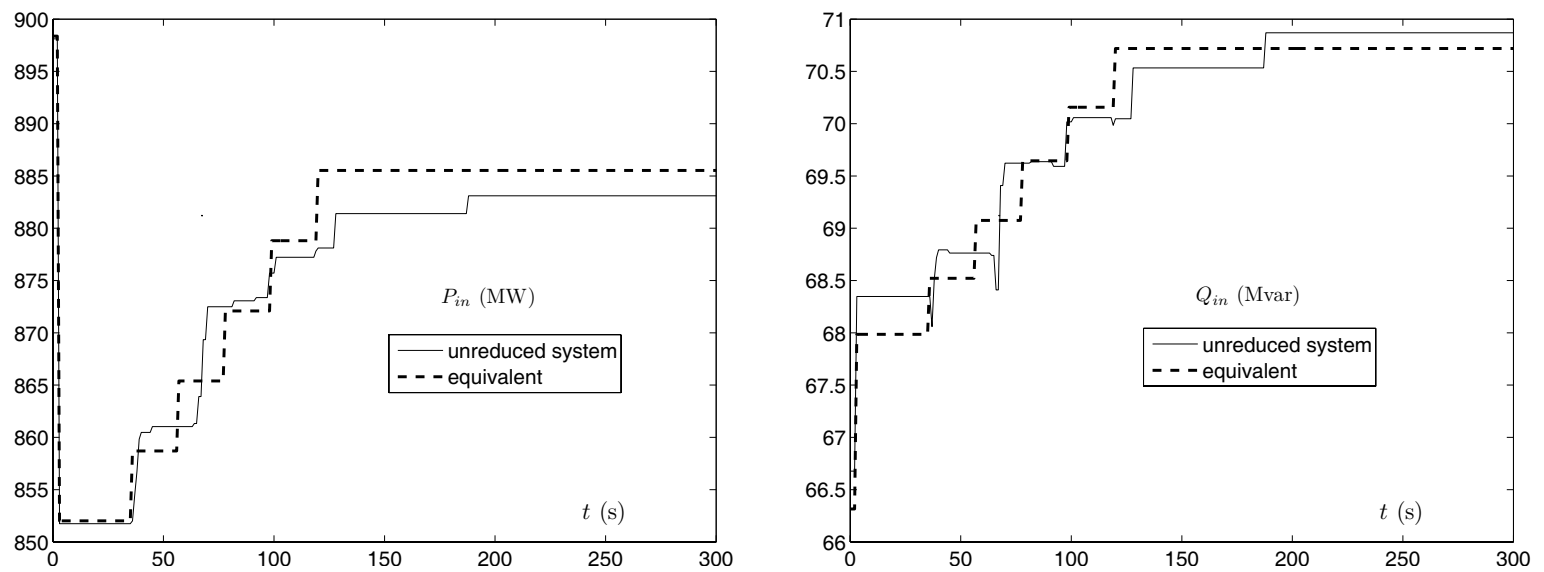

Fig. 8. active and reactive power evolution following a 0.04 pu voltage drop at the subtransmission feeder
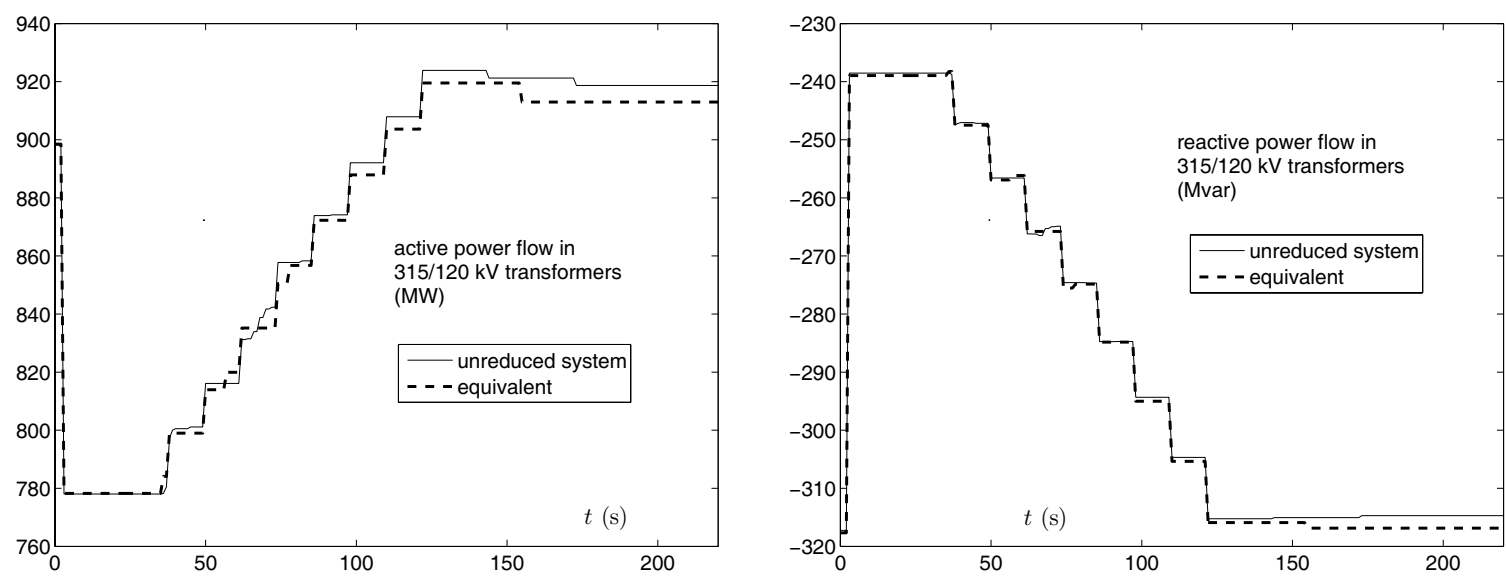

Fig. 9. active and reactive power evolution following a 0.1 pu voltage drop at the transmission bus; upper LTCs represented

shunt compensation present at the $120-\mathrm{kV}$ bus. Here too, the equivalent matches very well the original system.

Similar tests have been performed for various load models.
As an illustration, the responses in Fig. 10 correspond to constant current $\left(\alpha_{1}=\beta_{1}=1\right)$ loads. Expectedly, the shortterm drop in active power is less important than in Fig. 7, while 

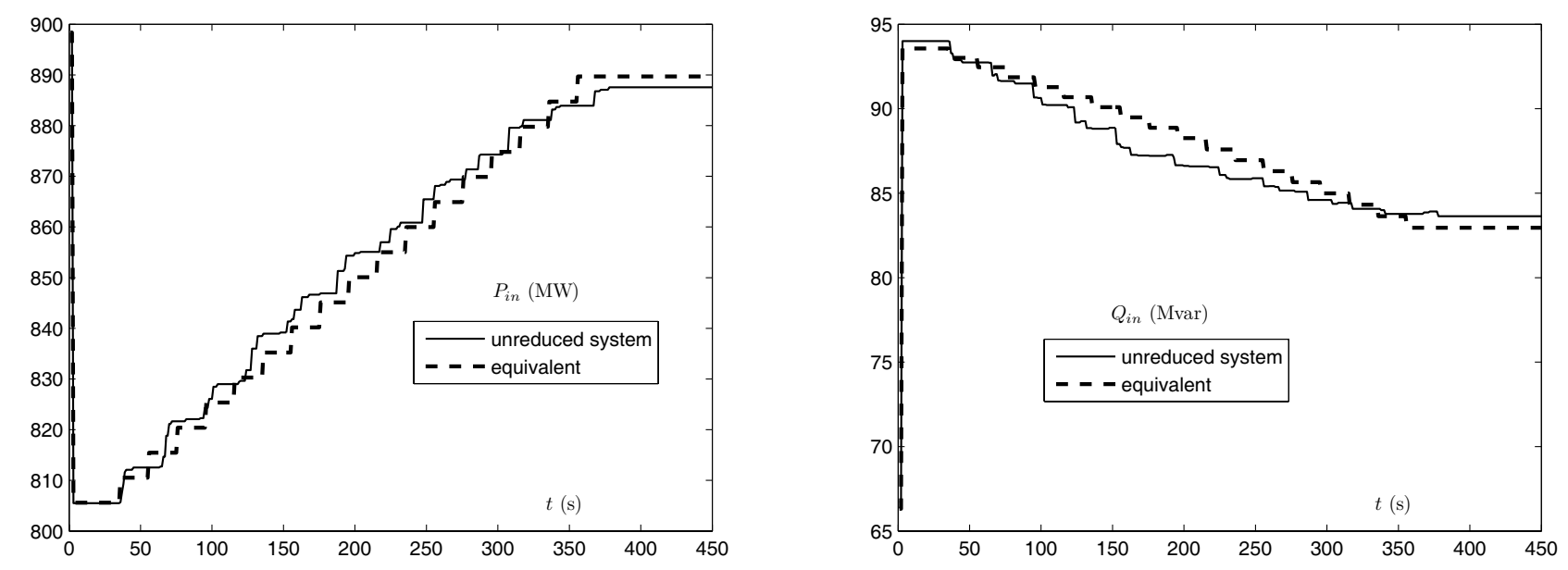

Fig. 10. active and reactive power evolution following a $0.1 \mathrm{pu}$ voltage drop at the transmission bus; constant current loads

the opposite holds for reactive power, the decrease in capacitor production being compensated by a lower load decrease. In both responses, the original system is matched accurately by the equivalent.

\section{CONCLUSion}

This paper has proposed a simple equivalent to account for the aggregate response of a set of loads fed through distribution transformers equipped with LTCs and connected through a subtransmission system. One of its features is that the original individual loads are collected at the same bus, thereby preserving their identity.

The proposed model is easily incorporated into standard dynamic simulation programs. For instance, the series admittance and the ideal transformer make up a standard transformer model, while the multi-exponential load representation is very common.

The preliminary tests reported in this paper have shown that the equivalencing procedure is accurate enough for practical applications. Other tests performed with other individual load models and on different Hydro-Québec sub-networks led us to the same conclusion.

Further investigations are carried out:

- to see how far the equivalent parameters can be kept constant when the initial load level changes

- to incorporate distributed generation with participation to voltage control

- to test the accuracy in the presence of dynamically modelled motor loads, and
- to extend the method to subtransmission systems with multiple entry points.

\section{ACKNOWLEDGEMENT}

The help of Anyck Dumas (TransEnergie division of HydroQuébec) on providing data and information is gratefully acknowledged.

\section{REFERENCES}

[1] C.W. Taylor, Power system voltage stability, EPRI Power System Engineering Series, Mc Graw-Hill, 1994

[2] T. Van Cutsem, C. Vournas, Voltage Stability of Electric Power Systems, Boston, Kluwer Academic Publishers, 1998

[3] IEEE Task Force on Load representation for dynamic performance, "Load Representation for Dynamic performance studies", IEEE Trans. on Power Systems, Vol. 8, No 2, pp. 472-482, May 1993

[4] IEEE Task Force on Load representation for dynamic performance, "Standard load models for power flow and dynamic performance simulation", IEEE Trans. on Power Systems, Vol. 10, No 3, pp. 1302-1313, 1995

[5] K. Morison, H. Hamadani, L. Wang, "Practical issues in load modelling for voltage stability studies", Proc. IEEE PES General Meeting, pp. 13921397, July 2003

[6] R. Lind, D. Karlsson, "Distribution System Modelling for voltage stability studies", IEEE Trans. on Power Systems, Vol. 11, No 4, pp. 1677-1682, 1996

[7] G.B. Denegri, M. Invernizzi, F. Milano, "Synthesis of an equivalent dynamic model for load areas with LTC transformers", Proc. IEEE PES General Meeting, pp. 641-646, 2001

[8] D.J. Hill, "Nonlinear load models with recovery for voltage stability studies", IEEE Trans. on Power Systems, Vol. 8, pp. 166-176, 1993

[9] D. Karlsson, D.J. Hill, "Modelling and identification of nonlinear dynamic loads in power systems", IEEE Trans. on Power Systems, Vol. 9, pp. 157166, 1994

[10] W. Xu, Y. Mansour, "Voltage stability analysis using generic dynamic load models", IEEE Trans. on Power Systems, Vol 9, pp. 479-493, 1994 